
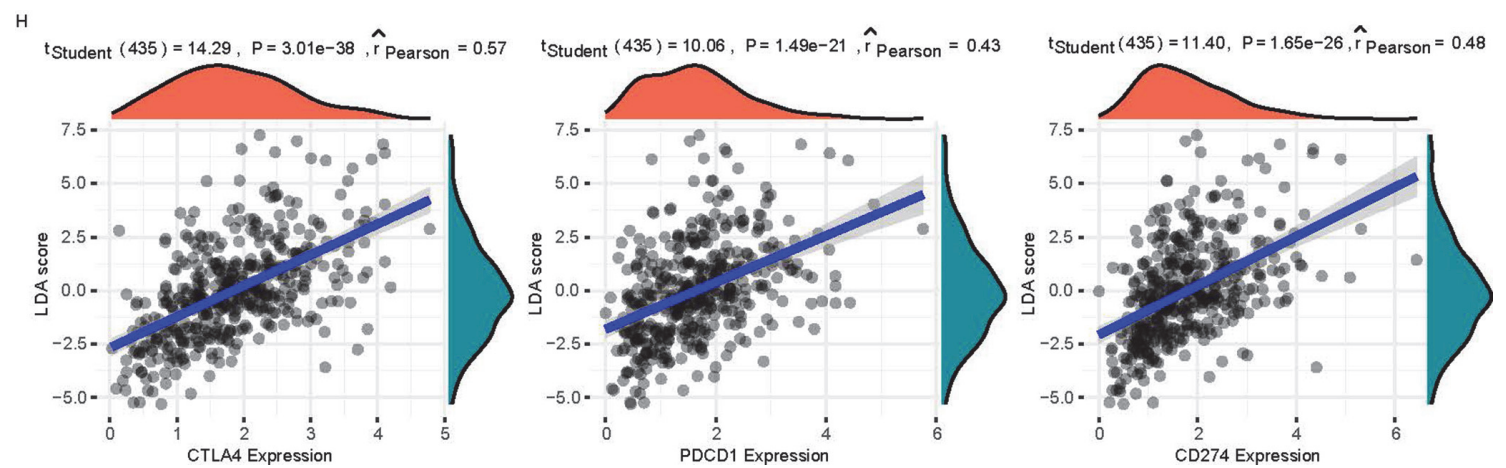

Abstract IDDF2021-ABS-0065 Figure 14
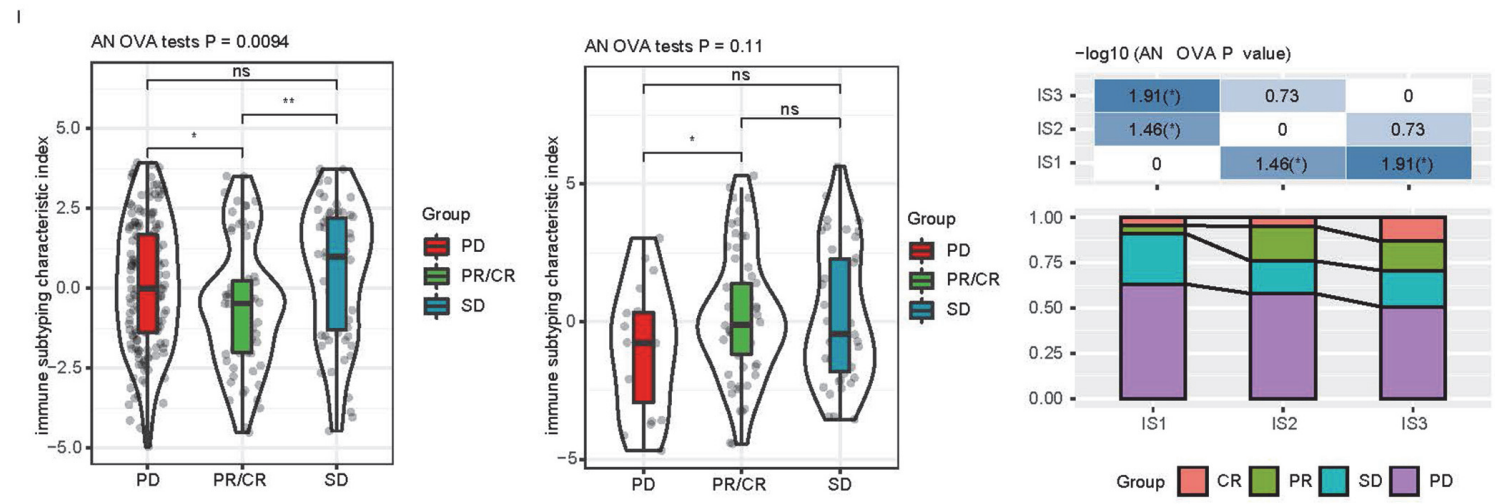

Abstract IDDF2021-ABS-0065 Figure 15

level of the partial response (PR)/complete response (CR) group is significantly lower than of stable (SD) and progressive disease (PD) group (IDDF2021-ABS-0065 Figure 15).

Conclusions The immune subtyping and ISCI system are indicative for the prediction of tumor prognosis of COADs. Identification of immune subtypes may facilitate the optimal selection of COAD patients responsive to adequate therapeutic strategies.

\section{IDDF2021-ABS-0066 ANTIOXIDANTS-RICH SUPPLEMENTS INCREASE FAECALIBACTERIUM ABUNDANCE IN GUT MICROBIOTA}

'Bangwei Chen*, ${ }^{2}$ Chuyao Wang, ${ }^{2}$ Zhiming Li, ${ }^{2}$ Tao Li. 'South China University of Technology, China; ${ }^{2} B G I-S h e n z h e n$, China

\subsection{6/gutjnl-2021-IDDF.27}

Background Antioxidants are becoming a popular type of dietary supplement. We believe a further understanding of the role of the gut microbiota in the health benefits of antioxidants could be gained from comparing the changes in gut microbiota composition and strain abundances from taking different sources of antioxidants.

Methods We recruited 120 volunteers and randomly divided them into 4 groups of 30 receiving grape seed extract (GSE), berries juice, vitamin $\mathrm{E}$ (VE), and amylodextrin (placebo). Stool samples were collected at baseline after 3 months of intervention, and after 3 months of washout period for metagenomic sequencing and analysis. Contig assembly and alignment were done using 4644 reference genomes to calculate relative abundance and annotate $\mathrm{KOs}$ for each strain. Associations between intervention, phases, and changes in bacterial relative abundance and functions were evaluated with paired Wilcoxon rank-sum test and McNemar's test for those strains with very low relative abundance.

Results We first analyzed the influence of the placebo vs. the whole antioxidants group. Shannon diversity and richness of the gut microbiota were not significantly different among the phases for both the placebo and the antioxidants group. Forty strains had significantly changed relative abundance in the antioxidants group $(\mathrm{p}<0.05)$, among which 13 were Faecalibacterium strains. Intervention-induced changes persisted after the washout period for a small number of strains. Then we investigated the similarities and differences among the three sources of antioxidants. Changes in relative abundances of butyric acid producers were found in both the juice and the VE group, while inflammation, tumor, and insulin resistancerelated bacteria Dorea formicigenerans, Fusobacterium nucleatum, and Enterobacter cloacae had lower, lower, and higher relative abundance in the GSE group. Furthermore, Faecalibacterium strains had higher relative abundance in all three groups.

Conclusions Through a long-term intervention study in humans, we found that although the sources of antioxidants did not alter the overall characteristics of the gut microbiota, each of them affected a unique set of bacteria strains, and the SCFA producer Faecalibacterium were increased in all three groups. 